NASA Technical Memorandum 4544

\title{
Multidisciplinary \\ Aeroelastic Analysis \\ of a Generic Hypersonic Vehicle
}

K.K. Gupta and K.L. Petersen

Dryden Flight Research Facility

Edwards, California

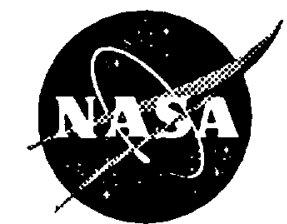

National Aeronautics and

Space Administration

Office of Management

Scientific and Technical Information Program 



\title{
MULTIDISCIPLINARY AEROELASTIC ANALYSIS OF A GENERIC HYPERSONIC VEHICLE
}

\author{
K. K. Gupta* \\ K. L. Petersen** \\ NASA Ames Research Center \\ Dryden Flight Research Facility \\ Edwands, CA
}

\begin{abstract}
This paper presents details of a flutter and stability analysis of aerospace structures such as hypersonic vehicles. Both structural and aerodynamic domains are discretized by the common finite element technique. A vibration analysis is first performed by the STARS code employing a block Lanczos solution scheme. This is followed by the generation of a linear aerodynamic grid for subsequent linear flutter analysis within subsonic and supersonic regimes of the flight envelope; the doublet lattice and constant pressure techniques are employed to generate the unsteady aerodynamic forces. Flutter analysis is then performed for several representative flight points. The nonlinear flutter solution is effected by first implementing a CFD solution of the entire vehicle. Thus, a 3-D unstructured grid for the entire flow domain is generated by a moving front technique. A finite element Euler solution is then implemented employing a quasiimplicit as well as an explicit solution scheme. A novel multidisciplinary analysis is next effected that employs modal and aerodynamic data to yield aerodynamic damping characteristics. Such analyses are performed for a number of flight points to yield a large set of pertinent data that define flight flutter characteristics of the vehicle. This paper outlines the finite-element-based integrated analysis procedures in detail, which is followed by the results of numerical analyses of flight flutter simulation.
\end{abstract}

\section{INTRODUCTION}

The accurate prediction of flight characteristics of hypersonic vehicles is vital during its design stage as well as prior to flight testing to ensure flight safety. Such a vehicle is expected to exhibit unprecedented levels of interaction among various disciplines such as structures, aerodynamics, and controls engineering, among others. For complex configurations, it is necessary to implement an efficient discretization procedure for effective and accurate idealization of the vehicle. In this connection, the finite element method proves to be a viable technique to model both solids and fluids continua and therefore is a natural choice for aeroelastic analysis that involves interaction of associated disciplines.
Multidisciplinary research at NASA Dryden Flight Research Facility is aimed at developing integrated aeroelastic and aeroservoelastic (ASE) analysis capabilities that can be conveniently employed for effective prediction of flight-critical, dynamic stability and control performance parameters and characteristics. A unified, nonlinear, multidisciplinary simulation analysis approach has been developed to accommodate these requirements, thus yielding a comprehensive analysis framework. The primary elements of this approach include 3-D unstructured fluids grid generation with adaptive mesh capabilities, finite element structural and heat transfer analysis procedures with advanced materials, as well as integrated aeroelastic and ASE analysis capabilities. Since such nonlinear analyses require extensive computing resources, much emphasis has been placed on developing novel solution schemes that reduce such solution effort significantly. Further, dedicated parallel processors have also been used to achieve the desired solution for practical problems within a reasonable span of time.

NASA's finite-element-based, multidisciplinary modeling and simulation computer program, STARS, ${ }^{1}$ developed for the solution of practical problems associated with NASA-critical flight projects, is described here in some detail. Extensive graphics-oriented pre- and postprocessing capabilities render the code useful for the solution of complex problems. The associated numerical formulations pertaining to various individual disciplines as well as the integrated solution techniques are discussed in the paper.

Some numerical examples are also presented to demonstrate the accuracy and efficacy of the NASA STARS code. The first example relates to a rectangular panel with clamped edges, and the STARS nonlinear flutter solution is compared with that obtained by linear analysis as well as wind-tunnel tests. A complete generic hypersonic vehicle is chosen next as an example problem. Relevant details of the structural modeling as well as a complete vibration analysis are presented, which constitute the first important step toward achieving an aeroelastic stability solution.

The results are presented for a set of parallel aeroelastic analyses pertaining to low Mach numbers within the flight flutuer envelope using linear, unsteady aerodynamic theory

\footnotetext{
- Member, AIAA

* Associate Fellow, AIAA
}

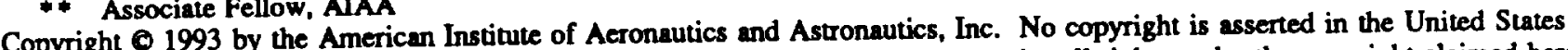
under Title 17, U.S. Code. The U.S. Government has a royalty-free license to exercise all rights under the copyright claimed herein for Governmental purposes. All other rights are reserved by the copyright owner. 
based on panel methods. This is followed by a number of CFD analyses pertaining to some representative flight conditions within the specified flight flutter envelope. These results are used in an attempt to generate unsteady aerodynamic forces and subsequently the damping characteristics that are indicative of aeroelastic instability.

\section{DESCRIPTION OF NUMERICAL TECHNIOUES}

To simulate nonlinear performance characteristics of advanced aerospace vehicles, it is necessary to integrate a number of relevant disciplines in a consistent fashion. A common employment of the finite element technique for the various disciplines such as the fluids and solids continua ensures accurate simulation of their interactions. The various finite element modules of the STARS program, developed in this connection, are shown in figure 1 , and some relevant details of their formulations are presented next.

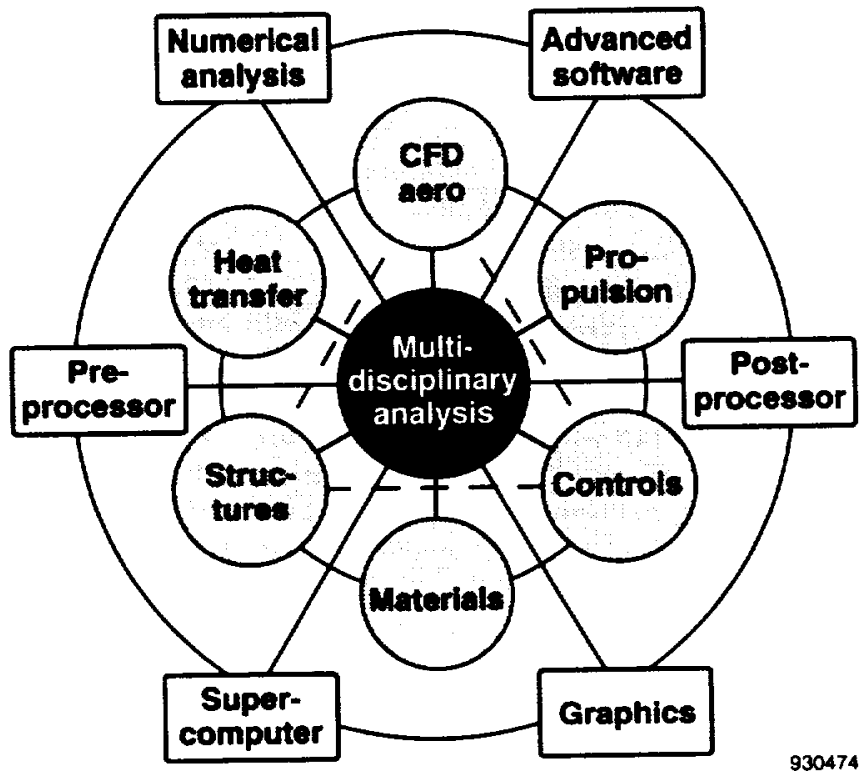

Fig. 1 STARS analysis modules.

Thus, the structural analysis module is capable of performing static, vibration, buckling, and dynamic response analyses of complex, practical structures having general, anisotropic material properties. A typical matrix formulation encompassing a broad class of structural problems may be written as

$$
M \ddot{u}+\left(C_{c}+C_{d}\right) \dot{u}+\left[K(1+i g)+K^{\prime}+K_{G}\right] \mathbf{u}=f(t)
$$

where

$$
\begin{aligned}
& \mathbf{M}=\text { inertia matrix } \\
& \mathbf{C}_{\mathrm{c}}, \mathrm{C}_{\mathrm{d}}=\text { Coriolis and viscous damping matriccs }
\end{aligned}
$$

$$
\begin{array}{ll}
\mathbf{K}, \mathbf{K}^{\prime}, \mathbf{K}_{\mathbf{G}} & =\begin{array}{l}
\text { elastic, centrifugal, and geometrical } \\
\text { stiffness matrices }
\end{array} \\
\mathbf{u} & =\text { unknown displacement vector } \\
\mathbf{g} & =\text { structural damping } \\
i & =\text { imaginary number, } \sqrt{-1} \\
\mathbf{f}(t) & =\begin{array}{l}
\text { externally-applied, time-dependent, forcing } \\
\text { function }
\end{array}
\end{array}
$$

The various matrices in equation (1) are usually large but sparse in form for complex practical problems, and the STARS numerical analysis module is designed to solve such matrix simultaneous equations and eigenvalue problems in an efficient fashion.

Layered, anisotropic, advanced composite finite elements are used for the design of advanced aerospacecraft. Related data for temperature-dependent properties are stored in the material module of the program.

The dynamic behavior of a viscous, heat-conducting, compressible fluid obeying conservation of mass, momentum, and energy may be expressed by a set of partial differential equations

$$
\frac{\partial V}{\partial t}+\frac{\partial F_{i}}{\partial x_{i}}=f_{b}, \quad i=1,2,3
$$

where the solution, flux, and body forces column vectors as well as the viscous stress tensor are defined as

$$
V=\left\{\begin{array}{lll}
\rho & \rho u_{j} & \rho E
\end{array}\right\}
$$

$$
\begin{gathered}
F_{i}=\left\{\begin{array}{c}
\rho u_{i} \rho u_{i} u_{j}+p \delta_{i j}+\sigma_{i j} \\
u_{i}(p+\rho E)+u_{1} \sigma_{i 1}+k \frac{\partial T}{\partial x_{i}}
\end{array}\right\} \\
f_{b}=\left\{\begin{array}{ll}
0 \quad f_{b j} \quad u_{1} f_{b 1}
\end{array}\right\} \\
\sigma_{i j}=-\frac{2}{3} \mu \frac{\partial u_{k}}{\partial x_{k}} \delta_{i j}+\mu\left(\frac{\partial u_{i}}{\partial x_{j}}+\frac{\partial u_{j}}{\partial x_{i}}\right)
\end{gathered}
$$

in which $\rho, p$, and $E$ are the density, average pressure intensity, and total energy, respectively; $\delta_{\mathrm{ij}}$ is the Kronecker delta; $u_{j}$ is the velocity component in the direction $x_{j}$ of a Cartesian coordinate system; $\mu$ is the viscosity; $k$ is the thermal conductivity; and $f_{b}$ represents body forces. The above equations are supplemented with the state equations

$$
p=(\gamma-1) p\left[E-\frac{1}{2} u_{i} u_{i}\right]
$$




$$
T=\left[E-\frac{1}{2} u_{i} u_{i}\right] c_{v}
$$

for a complete solution, in which $\gamma$ is the ratio of specific heats and $c_{v}$ is the specific heat at constant volume, such a formulation being valid for a perfect gas. A solution of the nonviscous form of equation (2) may be achieved by first obtaining a Taylor series expansion of $\mathbf{V}$ in time domain. The spatial domain, $\Omega$, is next discretized by unstructured meshes consisting of 3-D tetrahedron elements. Using linear, finite element approximations, $v=a \hat{v}, \hat{v}$ being nodal variable values, and employing a Galerkin weightedresidual procedure, a time-dependent form of the governing equations may be obtained as

$$
M \Delta \hat{v}=-\Delta t[c M+K] \hat{\mathbf{v}}+R
$$

in which $\mathbf{c}$ is a scalar, and $\mathbf{R}$ includes artificial viscosity effects essential for capturing shocks.

Equation (9) is solved by advancing the time-dependent form until steady conditions are obtained. Both an explicittimestepping, iterative scheme ${ }^{2}$ and a quasi-implicit solution algorithm have been incorporated in the STARS program to that effect. Thus, in the latter procedure, at a typical $n+1^{\text {th }}$ iteration stage, assuming the following relations,

$$
\begin{gathered}
\Delta \hat{\mathbf{V}}=\mathbf{V}_{n+1}-V_{n} \\
\hat{\mathbf{v}}=\left(\mathbf{V}_{n+1}-V_{n}\right) / 2
\end{gathered}
$$

equation (9) may be expressed as

$$
\begin{aligned}
& {\left[\left(1+\frac{\Delta t}{2} c\right) M+\frac{\Delta t}{2} K\right] V_{n+1}=} \\
& {\left[\left(1-\frac{\Delta t}{2} c\right) M-\frac{\Delta t}{2} K\right] V_{n}+R }
\end{aligned}
$$

or

$$
[A] V_{n+1}=[B] V_{n}+R
$$

Expressing

$$
\mathbf{A}=\mathbf{D}+\mathbf{O}
$$

in which $\mathbf{D}$ and $\mathbf{O}$ are respective matrices containing diagonal and off-diagonal terms of $\mathbf{A}$, the iterative solution procedure may be carried out as outer loop

$$
[D] \mathbf{v}_{n+1}=[B] \mathbf{v}_{n}-[O] \mathbf{v}_{n+1}+R
$$

Solve $\mathbf{V}_{\mathbf{n}+1}$ iteratively

inner loop

$$
\mathbf{v}_{\mathbf{n}+1}^{(\mathrm{i}+1)}=[\mathrm{D}]^{-1}\left([\mathrm{~B}] \mathbf{v}_{\mathbf{n}}-[\mathrm{O}] \mathbf{v}_{\mathbf{n}+1}^{(\mathrm{i})}+\mathbf{R}\right)
$$

If $V_{n+1}^{(i+1)} \neq V_{n+1}^{(i)}$, continue iteration

STOP

If $V_{n+1} \neq V_{n}$, go to $n+2$ step

STOP

Both solution schemes prove to be suitable for effective solution of practical problems.

The above solution techniques have been applied to a fluids domain idealized by unstructured grids. An advancing front technique, developed for automated 3-D mesh generation, 2 has been found to be suitable for discretization of complex domains. This procedure has been further modified ${ }^{3}$ to effect significant savings in solution time; this is achieved by first generating a grid whose cells have linear dimension about twice the required size, and then reducing each cell locally to reach desired cell sizes.

The STARS multidisciplinary analysis module performs aeroelastic and ASE analyses. For linear systems, ${ }^{4}$ the code uses panel methods to generate unsteady aerodynamic forces, and subsequent flutter analyses may be performed by either the $k, p-k$, or ASE method. A far more elaborate effort is required, however, for the nonlinear case in which the CFD technique yields the unsteady forces. Notable earlier efforts in this area have been based on the finite difference method, $5-7$ whereas our approach has been based entirely on the finite element technique. Figure 2 shows a flowchart of the major numerical solution steps adopted in the STARS program to perform nonlinear flutter analyses for complex aerospacecraft. References 3 and 8 give in detail related numerical formulations in which the generalized equations of motion are cast in state-space form from which a response solution can be achieved easily by using a standard procedure. A fast Fourier transform produces such response solution data to obtain the damping value, which is indicative of the degree of aeroelastic instability.

The generalized aerodynamic force vector, $\hat{\mathbf{f}}_{\mathrm{a}}$ (fig. 2 ), is assembled from nodal pressure values of the finite element structural grid which are in turn computed by interpolation 
from such values at aerodynamic grid points derived from a CFD Euler solution. In this process, for each structural node pertaining to an element, a triangular aerodynamic element encircling the node is first identified and its average nodal pressure value assigned to the node. This process is repeated for the rest of the nodes of the element and averaged among the nodes; such calculations are performed for all structural elements.

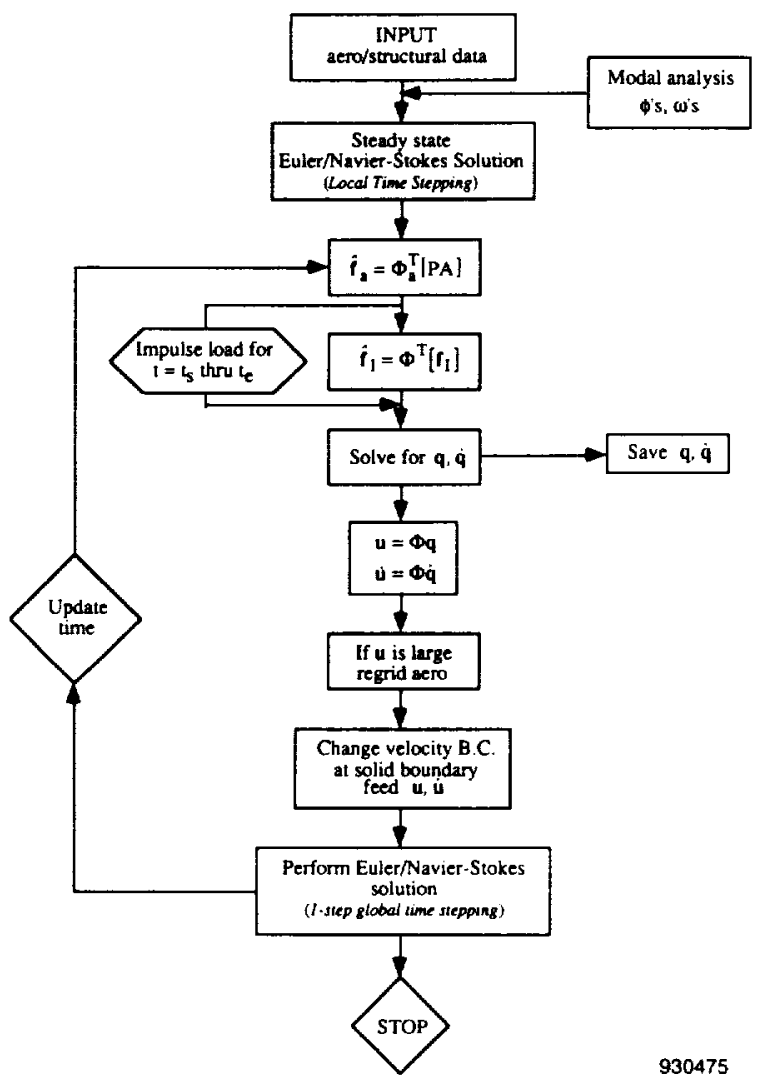

Fig. 2 Flowchart for nonlinear flutter analysis.

In the area of heat transfer analysis, the associated module can solve heat conduction problems for a wide array of structures, including composite ones. The program is capable of handling both linear and nonlincar radiation boundary conditions.

\section{NUMERICALEXAMPLES}

The STARS computer program is currently used to solve a large number of project-related problems at the NASA Dryden Flight Research Facility. Also, a large number of test cases has been solved by the program to assess the efficacy of solution algorithms and tools. Some relevant examples are presented here.

\section{Panel Flutter Analysis}

Reference 9 presents details of extensive flutter calculations for a rectangular panel employing approximate aerodynamic theory, and such results are also compared with experimental findings. The STARS program has been used to perform nonlinear flutter analysis of the panel with clamped edges, using the CFD and ASE modules of the code. Figure 3 shows the surface grid of the acrodynamic domain around a plate of aspect ratio 2 . Figure 4 depicts a comparison of flutter solutions by the various procedures; the flutter parameter is defined as $\lambda=$ $\frac{2 q a^{3}}{\beta D}$, in which

$\mathrm{q} \quad=$ airstream dynamic pressure
$\beta \quad=\sqrt{\mathrm{M}^{2}-1, \mathrm{M} \text { being the Mach number }}$
$\mathrm{D} \quad=\frac{\mathrm{Et}^{3}}{12\left(1-v^{2}\right)^{2}} \mathrm{E}, \mathrm{t}$, and $v$ being the elastic
$\begin{aligned} & \text { modulus, plate thickness, and Poisson's } \\ & \text { ratio, respectively }\end{aligned}$

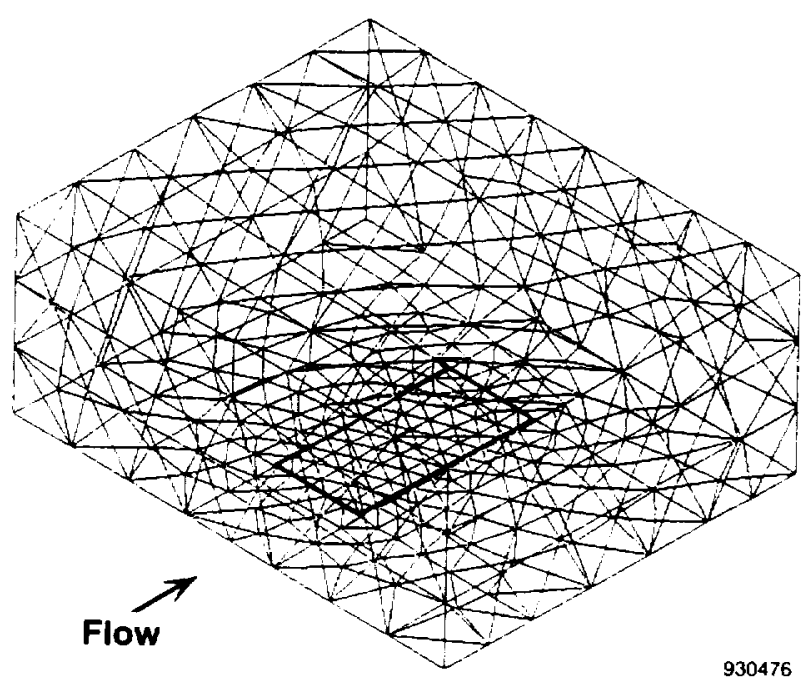

Fig. 3 Surface grid for aerodynamic domain for the clamped plate problem.

\section{Generic Hypersonic Vehicle Aeroelastic Analysis}

A new finite element structural model of the vehicle, upgraded from an earlier model, ${ }^{10}$ was recently generated in which special emphasis was placed on the generation of well-conditioned elements; the relevant numerical model has the following details:

$\begin{array}{lll}\text { No. of elements } & = & 4,990 \\ \text { No. of nodes } & = & 2,812 \\ \text { No. of degrees of freedom } & = & 16,872\end{array}$




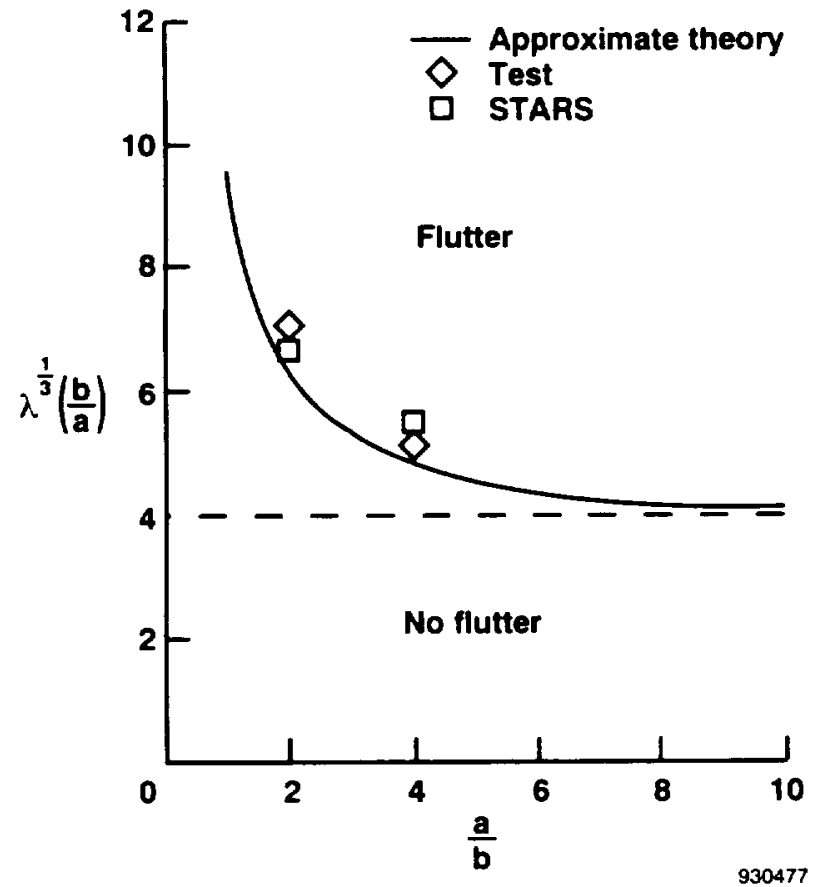

Fig. 4 Clamped rectangular panel - comparison of experimental, approximate aerodynamic theory and STARS nonlinear aeroelastic solution.

A free vibration analysis of the vehicle was performed by the STARS program using a block Lanczos algorithm; table I presents a summary of such results, and figure 5 shows some of the mode shapes.

Table I. Generic Hypersonic Vehiclc STARS free vibration analysis.

\begin{tabular}{|c|c|c|c|}
\hline Mode \# & Erequ & $\mathrm{acy}(\mathrm{Hz})$ & Description \\
\hline & STARS & $\mathrm{LaRC} / \mathrm{EAL}$ & \\
\hline $1-6$ & 0.0 & & rigid body modes \\
\hline 7 & 3.18 & 3.01 & fus. $1 b$, symm \\
\hline 8 & 4.03 & & wing $1 b$, anti \\
\hline 9 & 4.05 & 4.02 & wing $1 \mathrm{~b}$, symm \\
\hline 10 & 5.56 & & fus. $1 \mathrm{~b}$, anti \\
\hline 13 & 6.91 & 7.70 & wing $1 \mathrm{t}$, symm \\
\hline 14 & 6.97 & & wing $1 \mathrm{t}$, anti \\
\hline 15 & 7.27 & 7.06 & fus. $2 b$, symm \\
\hline 16 & 9.40 & 9.47 & fin $1 b$, symm \\
\hline 17 & 9.45 & & fin $1 b$, anti \\
\hline
\end{tabular}

Unsteady aerodynamic forces were next generated, using the linear aerodynamic module of STARS, employing panel methods. Figure 6 depicts the proposed flight envelope of the vehicle, also providing data on flutter analysis points, whereas figure 7 shows the vehicle linear aerodynamic paneling. Tables II and III give details of such analysis results.

Table II. Generic Hypersonic Vehicle - STARS symmetric half-aircraft flutter analysis.

$\begin{array}{llll}\text { Altitude (fi) } & \text { MachNo } & \text { Speed (KEAS) } & \text { Erea }(\mathrm{Hz}) \\ \text { Sea level } & 0.9 & 1807 & 5.5 \\ & 0.9 & 2273 & 3.3 \\ 10,000 & 0.9 & 1798 & 5.5 \\ & 0.9 & 2265 & 3.3 \\ 30,000 & 2.2 & 2286 & 5.4 \\ 50,000 & 2.2 & 2298 & 5.3 \\ & 3.4 & 2693 & 5.4 \\ 70,000 & 5.6 & 3347 & 5.6\end{array}$

Table III. Generic Hypersonic Vehicle - STARS antisymmetric half-aircraft flutter analysis.

\begin{tabular}{llll} 
Altitude (fi) & Mach No & Speed (KEAS) & Erea $(\mathrm{Hz})$ \\
\cline { 1 - 3 } Sca level & 0.9 & 1435 & 6.0 \\
& 0.9 & 1636 & 5.5 \\
10,000 & 0.9 & 1428 & 6.0 \\
& 0.9 & 1641 & 5.5 \\
30,000 & 2.2 & 1870 & 5.6 \\
& 2.2 & 2314 & 5.2 \\
50,000 & 2.2 & 1930 & 5.6 \\
& 2.2 & 2314 & 5.2 \\
& 3.4 & 2271 & 5.6 \\
& 3.4 & 2700 & 5.3
\end{tabular}

In an attempt to obtain nonlinear flutter solutions in the hypersonic flow regime, a set of Euler analyses were performed using the STARS CFD module. Thus, figure 8 shows some views of the associated unstructured acrodynamic grid that has the following details:

$$
\begin{array}{lll}
\text { No. of elements } & = & 262,787 \\
\text { No. of nodes } & = & 46,797
\end{array}
$$

The Euler analyses were performed for a number of flight conditions, and figures 9 and 10 show vehicle speed and pressure distribution for a typical flight condition, Mach = 5.6 and $\alpha=0$. 


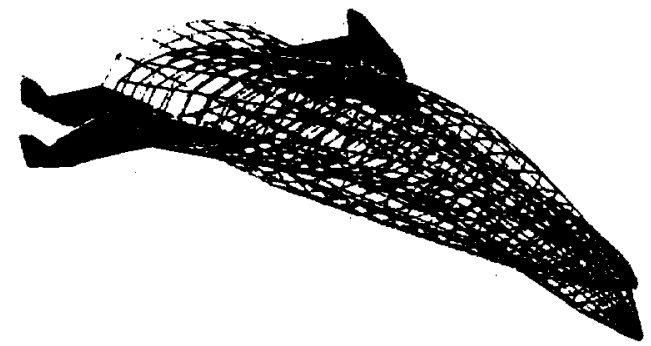

Fuselage 1st bending (S) $-3.18 \mathrm{~Hz}$

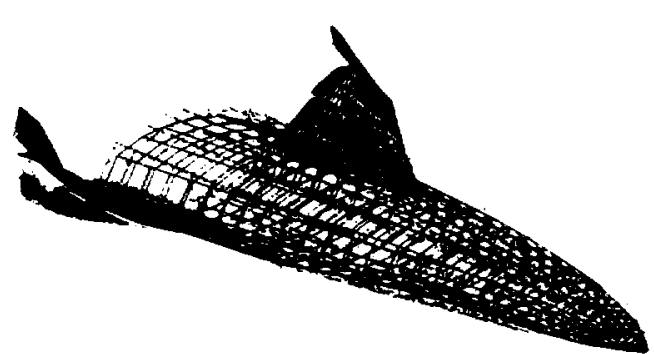

Wing 1st bending (S) $-4.05 \mathrm{~Hz}$

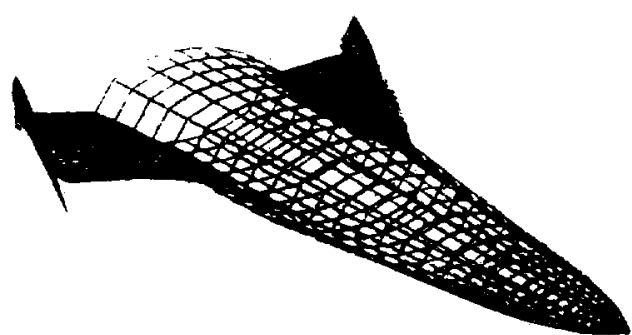

Wing 1st torsion (S) $-6.91 \mathrm{~Hz}$

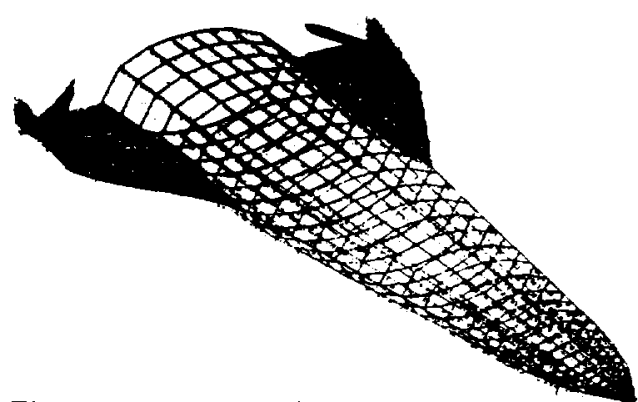

Fin 1st bending $(S)-9.40 \mathrm{~Hz}$

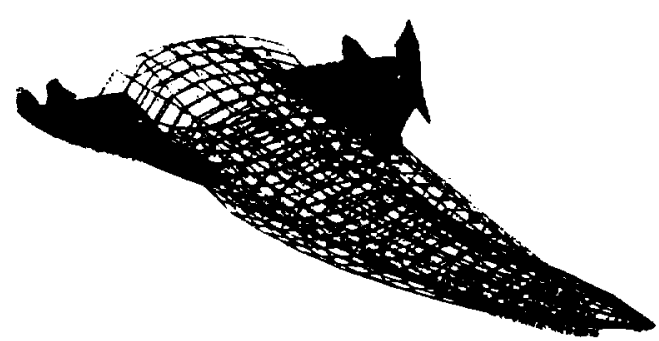

Fuselage 1st bending (A/S) $-5.56 \mathrm{~Hz}$

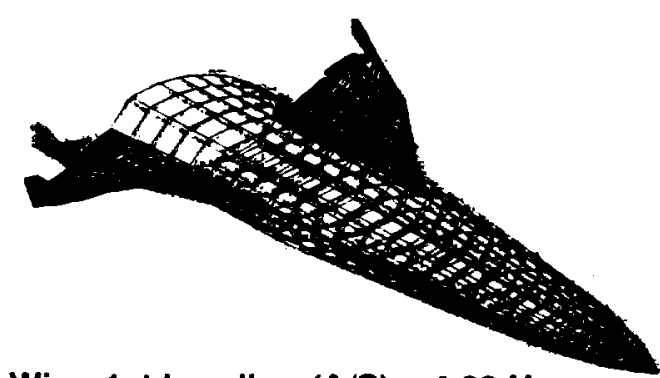

Wing 1st bending (A/S) $-4.03 \mathrm{~Hz}$

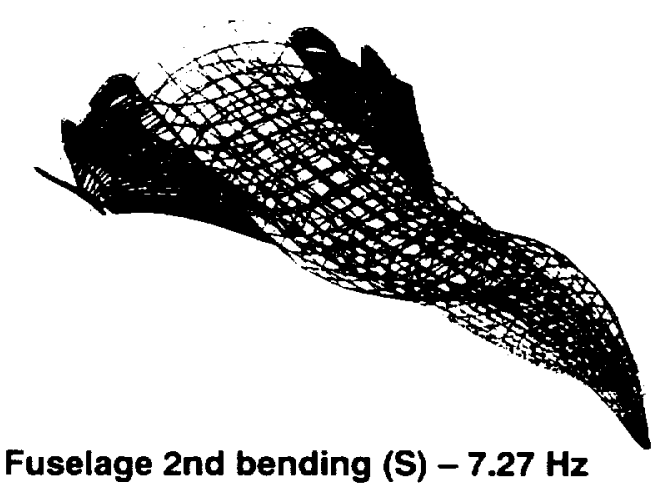

Fig. 5 Gencric Hypersonic Vehicle - some lypical mode shapes

(symmetric (S) and antisymmetric (A/S)). 


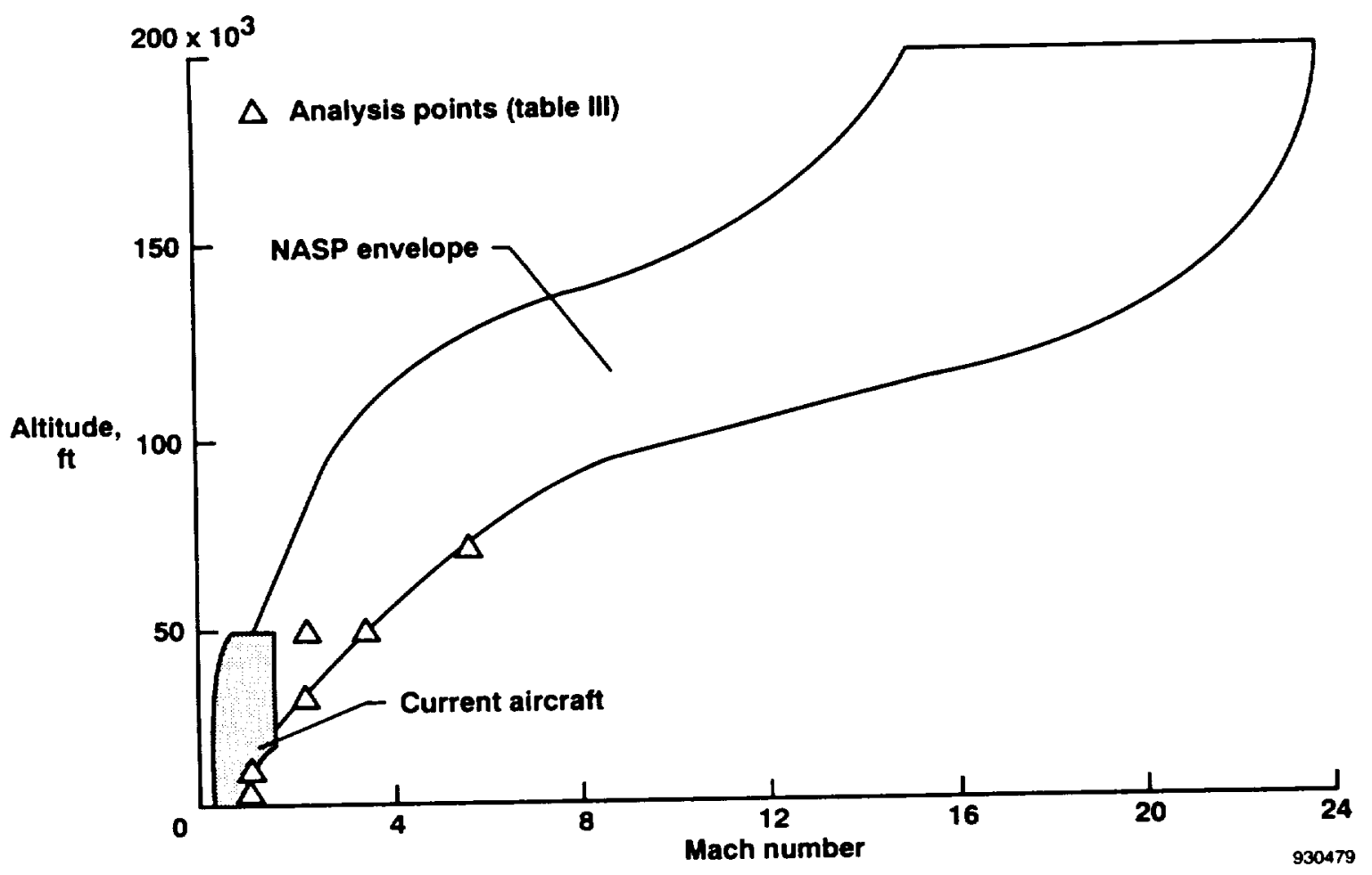

Fig. 6 Generic Hypersonic Vehicle - proposed flight envelope.

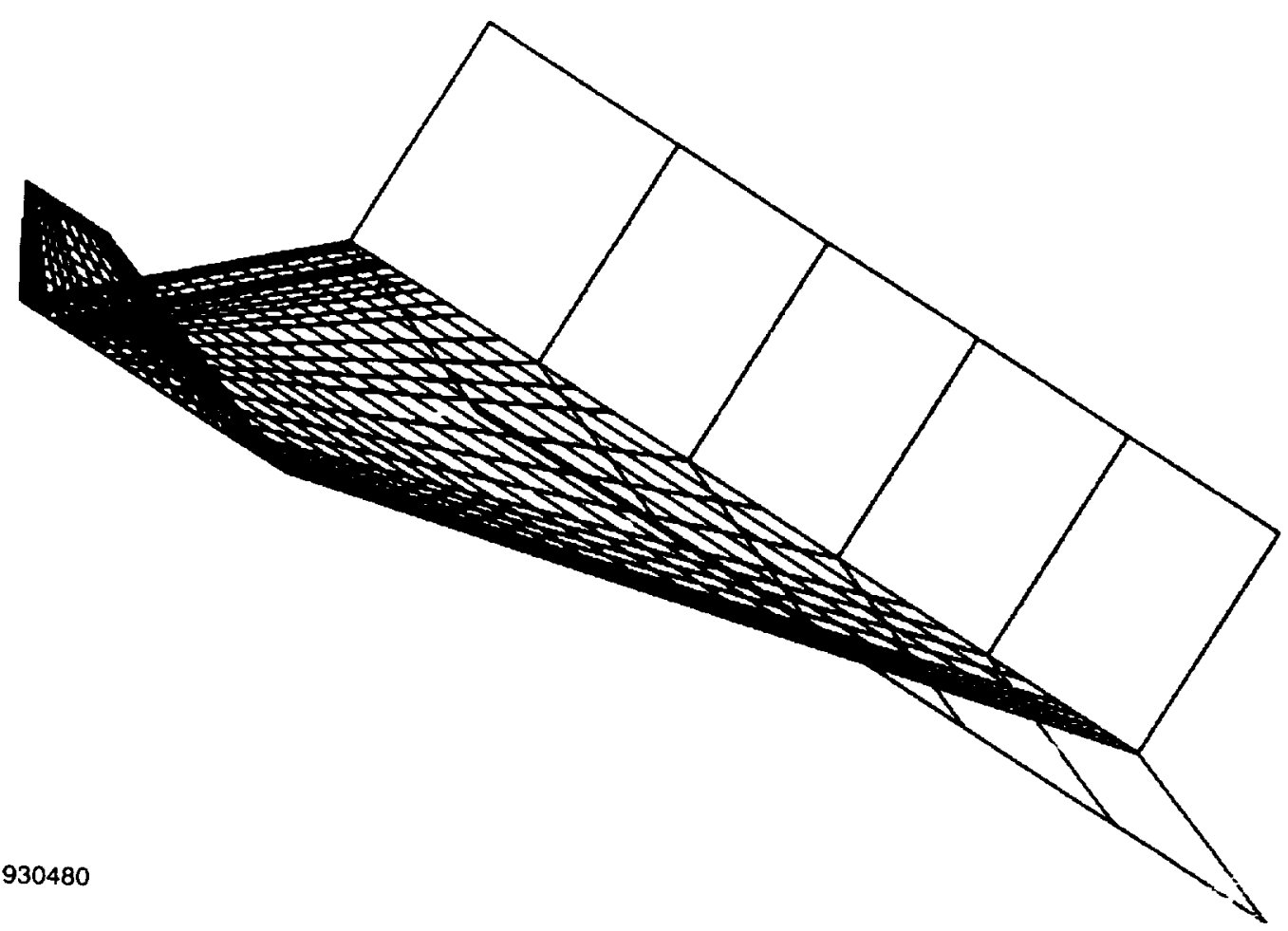

Fig. 7 Generic Hypersonic Vchicle - acrodynamic paneling for linear analysis. 


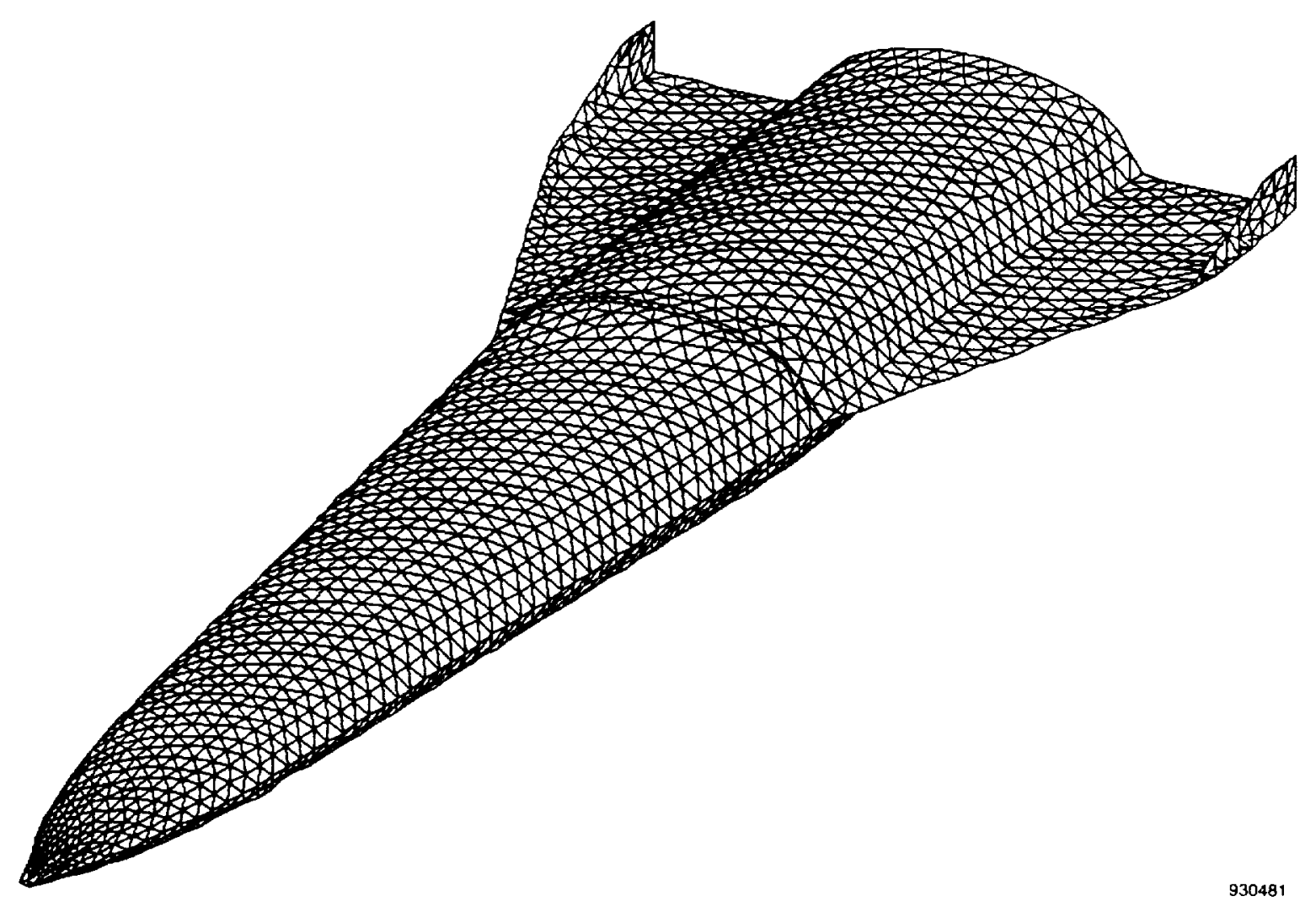

(a) Vehicle surface aerodynamic mesh.

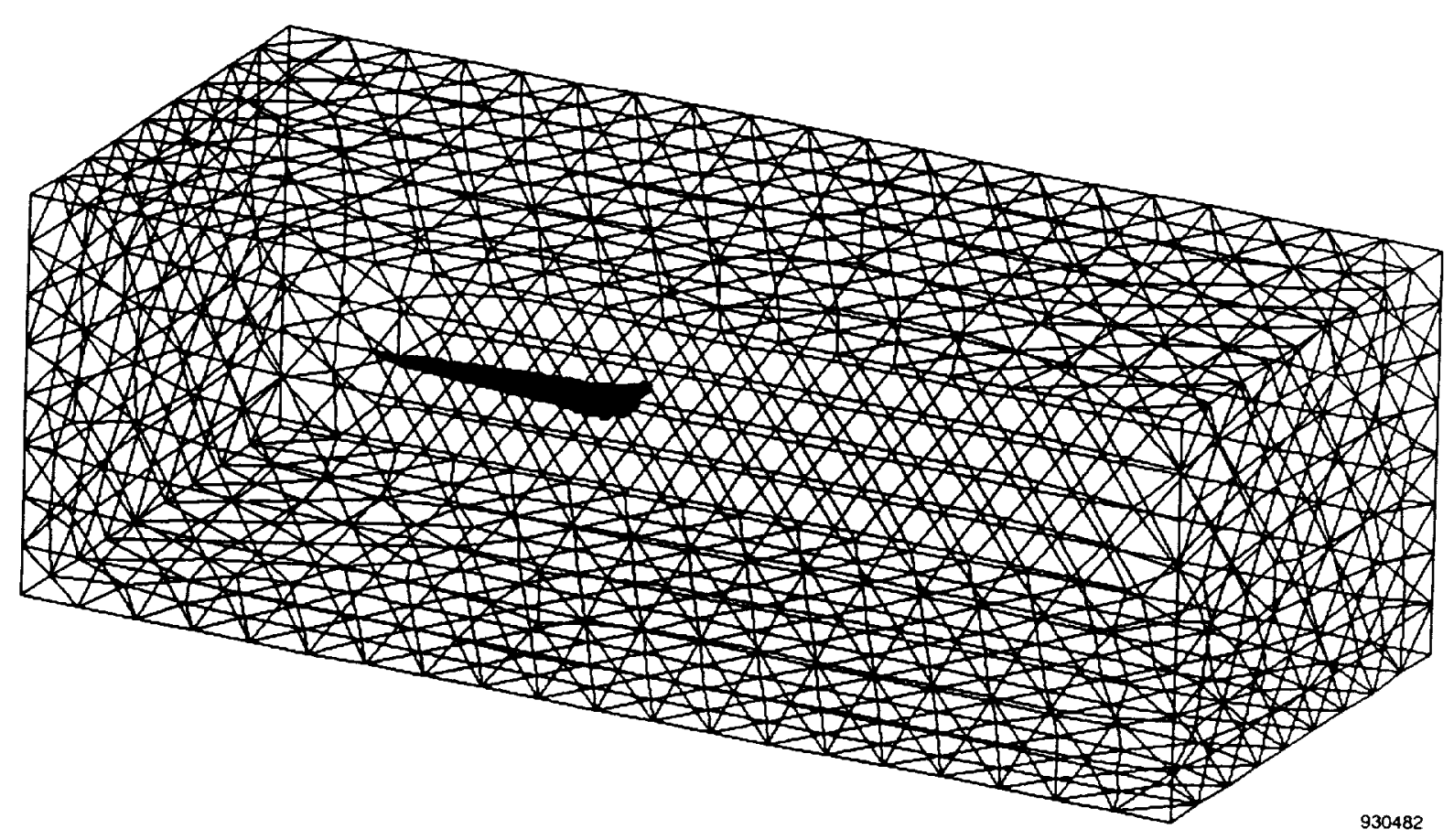

(b) Surface grid for 3-D solution domain.

Fig. 8 Generic Hypersonic Vehicle - complete surface mesh generation. 


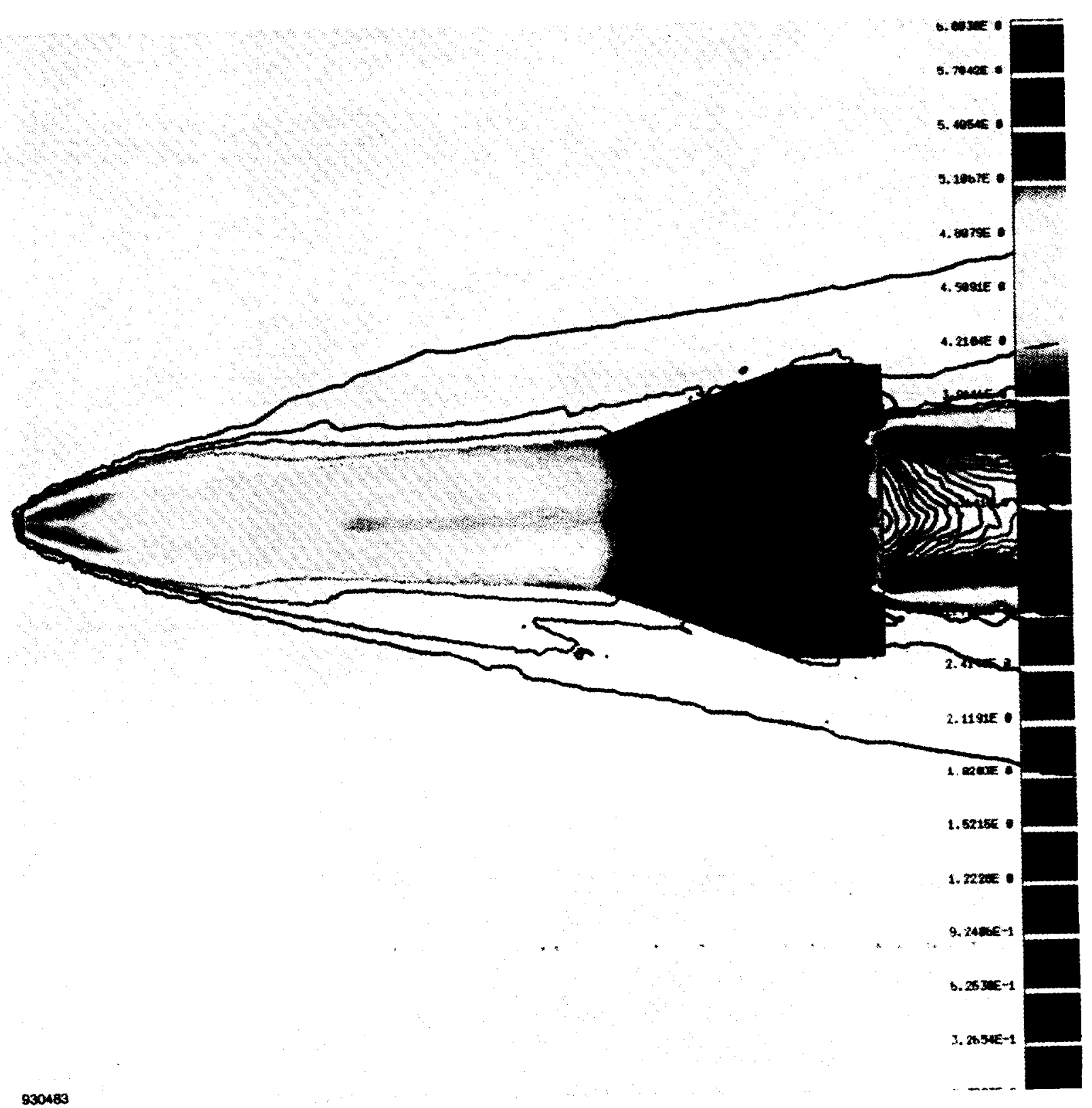

Fig. 9 Generic Hypersonic Vehicle (Mach = 5.6) - plan view of Mach number distribution. 


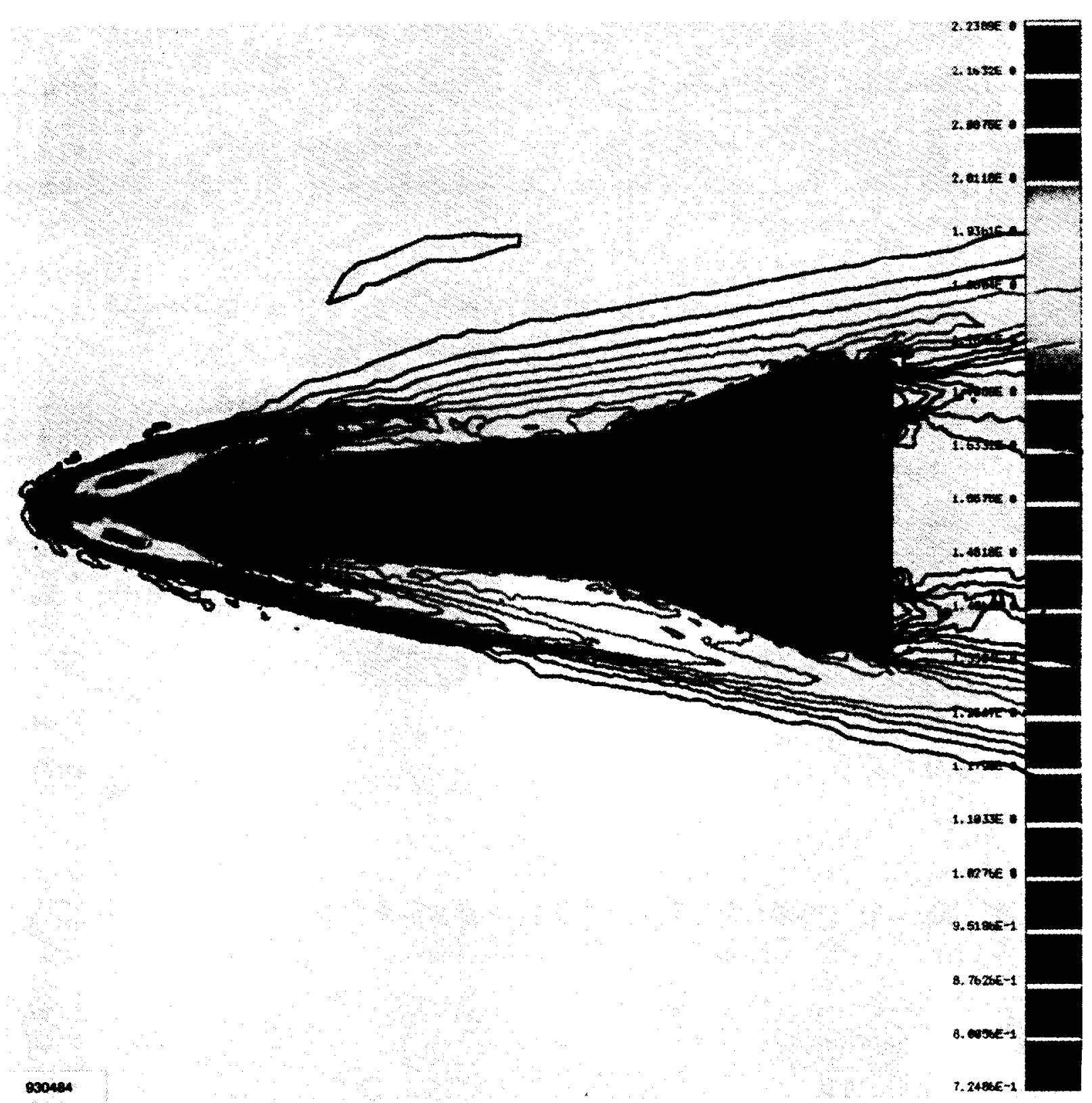

Fig. 10 Generic Hypersonic Vehicle (Mach = 7.0) - plan view of pressure distribution. 


\section{CONCLUDING REMARKS}

A finite-element-based analysis procedure developed for effective modeling and simulation of aerospacecraft that exhibit multidisciplinary interaction has been presented. Some numerical examples are also presented that demonstrate the applicability of the STARS computer program for the analysis of practical problems. Since both structural and fluids modeling are accomplished by the common finite element method, transfer of data between the two systems proves to be a natural process. Although a nonlinear aeroelastic analysis for a complete vehicle requires rather extensive computing resources, utilization of the current finite element-based procedure is justified in view of its ability to accurately model complex vehicle geometry.

\section{ACKNOWIEDGMENTS}

The authors are thankful to E. Hahn, R. Truax, T. Doyle, T. Walsh, C. Bach, and the other members of the STARS engineering group for their assistance in the preparation of the example problems. Also, assistance provided by $\mathrm{T}$. Walsh in the preparation of this manuscript is most appreciated.

\section{REEERENCES}

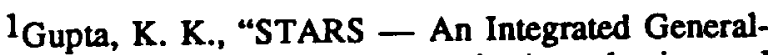
Purpose Finite Element Structural, Aeroelastic, and Aeroservoelastic Analysis Computer Program," NASA TM-101709, Jun. 1990.

2 Peraire, J., Peiro, J., Formaggia, L., Morgan, K., and Zienkiewicz, O. C., "Finite Element Euler Computations in Three Dimensions," Int. $j$. numer. methods eng., 26, 1988, pp. 2135-2159.
${ }^{3}$ Gupta, K. K., Petersen, K. L., and Lawson, C. L., "Multidisciplinary Modeling and Simulation of a Generic Hypersonic Vehicle," Paper No. AIAA-91-5015, AIAA 3rd International Aerospace Planes Conference, December 3-5. 1991, Orlando, FL.

${ }^{4}$ Gupta, K. K., Brenner, M. J., and Voelker, L. S., "Integrated Aeroservoelastic Analysis Capability with X29A Comparisons," J. Aircraft, 26, 1989, pp. 84-90.

5 Batina, John T., "Unsteady Euler Algorithm with Unstructured Dynamic Mesh for Complex-Aircraft Aerodynamic Analysis," AIAA Journal, 29, 1991, pp. 327-333.

${ }^{6}$ Batina, J. T., Lee, E. M., and Kleb, W. L., "Unstructured-Grid Methods Development for Unsteady Aerodynamic and Aeroelastic Analyses," in Proceedings of AGARD Structures and Materials Panel Specialists' Meeting on Transonic Unsteady Aerodynamics and Aeroelasticity, October 9-11, 1991, San Diego, CA. Also NASA TM-104143, Sep. 1991.

${ }^{7}$ Lee-Rausch, Elizabeth M., and Batina, John T., "Wing Flutter Boundary Prediction Using Unsteady Euler Aerodynamic Method," Paper No. AIAA-93-1422-CP. Also NASA TM-107732, Mar. 1993.

${ }^{8}$ Gupta, K. K., Petersen, K. L., and Lawson, C. L., "On Some Recent Advances in Multidisciplinary Analysis of Hypersonic Vehicles," Paper No. AIAA-92-5026, AlAA 4th International Aerospace Planes Conference, December $1-4,1992$, Orlando, FL.

${ }^{9}$ Dixon, Sidney C., "Comparison of Panel Flutter Results from Approximate Aerodynamic Theory with Results from Exact Inviscid Theory and Experiment," NASA TND-3649, Oct. 1966.

${ }^{10}$ Spain, C. V., Soistmann, D. L., and Linville, T. W., "Integration of Thermal Effects Into Finite Element Aerothermoelastic Analysis With Illustrative Results," NASA CR-1059, Aug. 1989. 
